ISSN electrónico: 2602-8069

\title{
UNA APROXIMACIÓN TEÓRICA AL CONSTRUCTO DE REPUTACIÓN
}

\section{A theoretical approach to the reputation construct}

\author{
Gabriela Baquerizo Neira \\ Universidad Casa Grande \\ gbaquerizo@casagrande.edu.ec \\ Marcela Pizarro \\ Universidad Austral \\ mpizarro@austral.edu.ar
}

Fecha de recepción del artículo: 07/09/2019

Fecha de aceptación definitiva: 11/11/2019 


\section{RESUMEN}

El estudio de la reputación en las últimas décadas ha tenido un incremento exponencial, debido a que profesionales y académicos de distintas disciplinas se han ocupado del tema. Esto no ha permitido que se llegue a un consenso entre los expertos y, por tanto, ha complejizado la comprensión de su definición y gestión. El objetivo de este artículo es describir el estado en el que se encuentra su estudio en los ámbitos corporativo y personal. A partir de la revisión de literatura en bases de datos como PROQUEST, EBSCO, DIALNET Y JSTOR, se obtienen teorías, conceptos, enfoques y reflexiones de los trabajos realizados hasta el momento. Como resultado de este estudio exploratorio se concluye que la reputación es de naturaleza perceptual y se construye mediante la acumulación temporal de comportamientos y acciones realizadas por la organización o el sujeto en un contexto determinado.

Palabras clave: Reputación, comunicación corporativa, teoría, management.

\section{ABSTRACT}

The study of reputation in recent decades has increased exponentially because professionals and academics from different disciplines have dealt with the subject. For this reason, experts have found it difficult to reach a consensus and to understand its definition and management. The aim of this article is to describe the state of their study in the corporate and personal spheres. From the review of literature in databases such as PROQUEST, EBSCO, DIALNET and JSTOR, theories, concepts, approaches and reflections are obtained from the work done so far. As a result of this exploratory study, it is concluded that reputation is perceptual in nature and is built through the temporary accumulation of behaviors and actions carried out by the organization or subject in a given context.

Keywords: Reputation, corporate communication, theory, management. 


\section{INTRODUCCIÓN}

\section{Problemáticas entorno al concepto de reputación}

En el sistema de intercambio que se vive a diario, la reputación constituye un elemento importante puesto que permite conocer si la gente con la que se establecen relaciones sociales o laborales son personas fiables, honestas y leales. Estar al tanto de cuáles son sus virtudes y sus defectos; conocer con quién se va a tratar e intuir qué trato vamos a recibir; conocer cuáles son sus aptitudes e identificar en qué lugar deben ubicarse dentro del sistema para que exploten al máximo sus habilidades, son factores elementales para la convivencia, el desarrollo organizacional y social.

A pesar de la abundante bibliografía existente alrededor del tema, y de la diversidad de enfoques desde donde se lo estudia, no existe una noción universal de lo que es la reputación. Asimismo, al ser este un campo de estudio multidisciplinario no es posible lograr un acuerdo sobre su constructo, ${ }^{1}$ lo que dificulta —en muchos casos-su definición.

Expertos en el tema, ${ }^{2}$ comprenden a la reputación como un fenómeno de naturaleza multidimensional que contribuye a generar valor positivo o negativo en una persona, un objeto o una organización. Es "el juicio que merecemos al concepto público" y juega un papel importante en la relación que se establece entre las personas y las organizaciones (Barcia, 1894, 687).

Según los argumentos de Emler (1990, 172), la opinión acerca de una persona, es decir, la reputación es un concepto "exclusivamente humano, debido a la capacidad del hombre para hablar y el proceso social que el lenguaje le permite vivir. Esto hace que las personas tengan reputación solo en la medida en que se relacionan con los demás". Que se forja como resultado de una correspondencia positiva entre la participación social del individuo y la construcción de sus actos y comportamientos; porque las actuaciones públicas son las que provocan las impresiones en las audiencias y pueden mantenerse en el tiempo y ser gestionadas por las personas otorgándoles reconocimiento por parte de los demás.

Barcia señala que es una de las "voces más sabias que tiene nuestra lengua":

Reputación, nombre (...) viene del latín puto, putas, putare, putavi, putatum, que equivale a juzgar; es decir, a sentenciar con el entendimiento. Y como nuestra fama ó nuestro crédito personal es una cosa que se juzga todos los días por el público, se añadió á putare la partícula re, que significa reiteración, y así se formó reputar. Reputar, pues, es juzgar repetidamente a una persona; ó volviendo a la idea anterior, es sentenciarla todos los días ante el tribunal de la moral pública. Por consecuencia, reputación no significa sino el juicio que merecemos al concepto público (Barcia, 1882, 678).

1 Se entiende en este trabajo por constructo a la construcción (teórica) hipotética. Esto es, siguiendo a Dorsch, un concepto operacional de aprehensión inmediata que hace referencia a entidades o cualidades no observables o detectables directamente. No son definibles en el lenguaje de la observación pura, se introducen a través de postulados y muchas veces quedan sin aclarar del todo. No son conjeturas formuladas al azar, sino que se derivan de un contexto teórico y con ayuda de procesos observables (Dorsch, 1994, 156).

2 Bromley, 2001; Davies, G., Chun, R., da Silva, R. V., y Roper, S., 2004; Dollinger, M. J., Golden, P. A., y Saxton, T., 1997; Fombrun y Van Riel, 1997; Fombrun y Rindova, 2000; Gotsi y Wilson, 2001; Ruiz, Gutiérrez y Águeda, 2012; Walker, 2010. 
Siguiendo al mismo autor, este juicio puede ser positivo o negativo. Además, manifiesta que la reputación "nos da a conocer como seres morales (...) nos sentencia: se refiere a la sociedad" (Barcia, 1882:678). Por consiguiente, se puede decir que la reputación es inherente al ser humano, ya sea porque se la atribuyen los demás o por la opinión de sí mismo que se otorga el individuo, aunque esta no vaya en consonancia con lo que opinan sobre él los otros.

A partir de una amplia revisión de literatura en bases de datos como PROQUEST, EBSCO, DIALNET y JSTOR, se exponen las diversas concepciones teóricas que servirán de base para nuestra investigación y que se detallan a continuación.

\section{DESARROLLO}

\section{Hacia un concepto de reputación}

En el mundo contemporáneo, el concepto de reputación se ha estudiado en muy diversas disciplinas: economía, contabilidad, administración, marketing, estrategia y sociología (e. g., Aula y Heinonen, 2005; Chun, 2005; Davies et al., 2004; Fombrun y Shanley, 1990; Newmark, 2011). Esta variedad de enfoques determina no solo la ausencia de un consenso en su definición, sino también que exista una cierta confusión entre su concepto y otros términos relacionados con ella como, por ejemplo: identidad, imagen, legitimidad, prestigio, renombre, estatus, buena voluntad, estima, posición, personalidad y cultura.

Estudiosos del tema coinciden con lo expuesto y sostienen que sí existe un acuerdo generalizado en que la principal barrera para llegar a un concepto universal de la reputación es la variedad de áreas temáticas que han abordado su estudio (e.g., Mahon, 2002; Walker, 2010).

Desde las escuelas de negocios y administración de empresas se ha indagado mucho acerca del tema y su relación con diversos elementos de la organización. Un ejemplo de ello son Michael Barnett, John Jermier y Barbara Lafferty (2006), investigadores de la Escuela de Administración de la Universidad del Sur de Florida que analizaron 49 fuentes únicas entre artículos y libros y coinciden en que son tres los conceptos principales que conforman la reputación, a saber: a) conocimiento, b) evaluación, y c) activo (ver Tabla 1). Después de una amplia revisión de literatura, estos autores concluyen que, a pesar de la inexistencia de una definición académica común, la reputación es el reflejo de una evaluación o un juicio.

Tabla 1. Conceptos generadores de la reputación

\begin{tabular}{|l|l|l|}
\hline \multicolumn{1}{|c|}{ Conocimiento } & \multicolumn{1}{|c|}{ Evaluación } & \multicolumn{1}{c|}{ Activo } \\
\hline $\begin{array}{l}\text { Información general que los ob- } \\
\text { servadores o públicos con los } \\
\text { que se relaciona la corporación } \\
\text { poseen sobre la empresa. }\end{array}$ & $\begin{array}{l}\text { Apreciación que los públicos tie- } \\
\text { nen sobre la organización. Jui- } \\
\text { cios, estimación y medición de la } \\
\text { institución. }\end{array}$ & $\begin{array}{l}\text { Recursos que agregan valor a la } \\
\text { organización como: intangible, } \\
\text { financiero o económico. }\end{array}$ \\
\hline
\end{tabular}

Fuente: Elaboración propia a partir de la propuesta de Barnett et al., 2006.

Otra revisión sistemática sobre reputación corporativa es la que realiza Kent Walker (2010, 358-359), especialista en RSC ${ }^{3}$ y desarrollo sostenible. En su estudio, identifica, en primer lugar, 1559 artículos en diversas revistas especializadas con publicaciones de alta

3 Responsabilidad social corporativa. 
calidad (e.g., Corporate Reputation Review; Academy of Management Review; Journal of Marketing; Business \&Society; entre otras), teniendo en cuenta los siguientes elementos: 1. palabra clave y término de identificación; 2. identificación del artículo; 3 . calidad de la evaluación; 4. extracción de la data; y 5. síntesis de la data. En segundo lugar, y a partir de lo encontrado, realiza una selección de las publicaciones en función del interés de su investigación, que se centra en analizar tres problemas fundamentales que existen en la literatura sobre reputación: 1 . La carencia de una definición clara y aceptada; 2 . La dificultad de operacionalizar la reputación corporativa; y 3 . La necesidad de una teoría más desarrollada sobre el tema.

Después de la revisión literaria, Walker (2010) concluye que solamente 43 artículos examinan la reputación corporativa: dos se centran en el valor de marca, seis en identidad organizacional y tres en marca corporativa. Así, este autor construye una tabla en la que 19 artículos (de los 43) proponen una definición de reputación en consonancia con la elaborada por Fombrun (1996).

Charles Fombrun (1996), creador de sistemas de gestión de reputación y experto en el tema, define la reputación como: 1 . El producto de una construcción social; 2 . Un conjunto de percepciones; y, 3. El resultado de la comparación entre percepciones.

Así, tomando el planteamiento de Fombrun (1996) y los hallazgos de la revisión documental de los 43 artículos, Walker incorpora dos elementos más a la concepción de reputación, a saber: a) que puede ser positiva o negativa y, b) que puede ser estable y duradera. De este modo, basándose en los cinco elementos anteriores define a la reputación corporativa como: "Un problema específico de representación perceptual relativamente estable, basado en las acciones pasadas de una empresa y las perspectivas de futuro, comparadas con algún estándar de calidad" (Walker, 2010, 370).

También es conveniente señalar que, de la revisión bibliográfica sobre reputación corporativa, una de las barreras más importantes para establecer una sola definición es la confusión que existe entre los conceptos de identidad (e.g., construcción social); imagen (e.g., percepciones); y reputación (Barnett et al., 2006). Aunque en este trabajo de investigación el tratamiento que se da a estos conceptos es divergente, algunos estudiosos aún los refieren como intercambiables (e.g. Brown et al., 2006; Wartick, 2002; Whetten y Mackey, 2002). Asimismo, se puede concluir que la reputación corporativa es considerada como el producto de una construcción social, que puede ser positiva o negativa y que es el resultado de la acumulación de las acciones de la organización a lo largo del tiempo.

En cuanto a la reputación personal, la gran mayoría de los estudios se han realizado desde el enfoque del comportamiento organizacional (Ferris, G. R., Blass, F. R., Douglas, C., Kolodinsky, R. W., Treadway, D. C., y Greenburg, J., 2003; Zinko et al., 2012). Los expertos que han profundizado en el tema concluyen que, el sujeto es un elemento que influye en la reputación de la organización y que la reputación del individuo no puede estudiarse fuera de un contexto y una temporalidad.

A continuación, se profundiza el análisis en las principales tradiciones teóricas desde las que se han estudiado tanto la reputación corporativa como la reputación personal.

4 Todas las citas textuales en inglés están traducidas al español por las autoras de este trabajo. 


\section{Principales áreas, enfoques y autores que han estudiado la reputacióncorporativa}

Como ya se expuso en líneas anteriores, la reputación es un constructo multidimensional que carece de una definición consensuada por la diversidad de disciplinas que han abordado su estudio. Sin embargo, la escuela norteamericana tiene una vasta trayectoria de investigación referente al tema, sobre todo en el ámbito corporativo.

De acuerdo con Ferris et al. (2014) no existe una teoría de la reputación. Sugieren que su análisis se plantea desde diversos postulados como: 1. teoría de señales (signalling theory); 2. teoría de la comparación social (social comparison theory); y 3. teoría de la influencia social y gestión de impresiones (social influence and impression management theory).

A estos marcos conceptuales, Fombrun (2012) agrega los siguientes, como doctrinas que también han influido en la conceptualización de la reputación corporativa: a) teoría institucional (institutional theory); b) teoría de los recursos y capacidades de la firma (resource based view theory - RBV-); c) teoría de la configuración de la agenda (agenda setting theory); d) teoría de los públicos (stakeholder theory); e) teoría de la identidad (identity theory); y f) teoría de la construcción social (social construction theory). Asimismo, Jensen, M., Kim, H., y Kim, B. K. (2012) incorporan —desde un enfoque psicosocial— la teoría de roles (role theory) al estudio de la reputación personal.

Con el análisis precedente también coincide Walker (2010) quien, a partir de una extensa revisión de trabajos previos, identifica que las teorías más utilizadas para la investigación de la reputación corporativa son: 1. teoría institucional (institutional theory); 2. teoría de señales (signalling theory); y 3. teoría de los recursos y capacidades de la firma (resource based view theory - RBV—). Este mismo autor, al igual que los ya mencionados, concluyen que debido al carácter multidimensional de la reputación no existe un consenso científico pues concurren múltiples percepciones que surgen según los públicos con los que se relaciona la organización.

Así, las definiciones del concepto reputación son muchas y variadas porque se originan en función del ámbito de estudio que aborda la problemática. A continuación, se exponen - a manera de resumen - las propuestas del concepto de reputación que realizan autores como: Mahon (2002), Rindova, V. P., Williamson, I. O., Petkova, A. P., y Sever, J. M. (2005) y Fombrun (2012), a partir del análisis de varias publicaciones que indagan sobre el fenómeno de la construcción de la reputación.

Tabla 2. Definiciones de reputación

\begin{tabular}{|l|l|l|}
\hline \multicolumn{1}{|c|}{ Referencia (Autores) } & \multicolumn{1}{|c|}{ Conceptos y palabras clave } & \multicolumn{1}{c|}{ Principales hallazgos } \\
\hline $\begin{array}{l}\text { Barnett et al. (2006) } \\
\text { Walker (2010) }\end{array}$ & Definición de la Reputación & $\begin{array}{l}\text { La reputación corporativa es un } \\
\text { juicio colectivo acerca de una orga- } \\
\text { nización basada en la evaluación } \\
\text { de su impacto financiero, social y } \\
\text { ambiental en el tiempo. }\end{array}$ \\
\hline Albert \& Whetten (1985) & $\begin{array}{l}\text { Identity Theory (teoría de la } \\
\text { identidad) }\end{array}$ & $\begin{array}{l}\text { La identidad organizacional describe } \\
\text { las características centrales de la } \\
\text { compañía, que son distintivas y } \\
\text { permanentes. }\end{array}$ \\
\hline
\end{tabular}




\begin{tabular}{|c|c|c|}
\hline $\begin{array}{l}\text { Spence (1974) } \\
\text { Schlenker (1980) } \\
\text { Lang \& Lang (1988) } \\
\text { Weigelt \& Camerer (1988) } \\
\text { Raub y Weesie (1990) } \\
\text { Dutton and Dukerich (1991) } \\
\text { Camic (1992) } \\
\text { Bromley (1993) } \\
\text { Yoon et al. (1994) } \\
\text { Dutton et al. (1994) } \\
\text { Kollock (1994) } \\
\text { Stephens y Greer (1995) } \\
\text { Gioia and Thomas (1996) } \\
\text { Hayward y Boeker (1998) } \\
\text { Montagliani y Giacalone (1998) } \\
\text { Cambell (1999) } \\
\text { Weiss, Anderson y Macinnis (1999) } \\
\text { Stuart (2000) } \\
\text { Prabhu y Stewart (2001) }\end{array}$ & $\begin{array}{l}\text { Signaling/ Impressions Theory (teo- } \\
\text { ría de las impresiones) } \\
\text { Game Theory (teoría de juegos) }\end{array}$ & $\begin{array}{l}\text { Las empresas señalan sus carac- } \\
\text { terísticas con el fin de influir en el } \\
\text { comportamiento de la competencia } \\
\text { y los stakeholders. La reputación se } \\
\text { atribuye a una empresa de acuerdo } \\
\text { con sus acciones pasadas. }\end{array}$ \\
\hline $\begin{array}{l}\text { Carroll y McCombs (2003) } \\
\text { Carroll (2010) }\end{array}$ & $\begin{array}{l}\text { Agenda Setting Theory } \\
\text { (teoría del establecimiento de la } \\
\text { agenda) }\end{array}$ & $\begin{array}{l}\text { Los medios de comunicación influ- } \\
\text { yen en las percepciones que tienen } \\
\text { los públicos sobre las empresas. } \\
\text { Afectan su visibilidad porque deter- } \\
\text { minan las características que deben } \\
\text { asociar los consumidores con ellas. }\end{array}$ \\
\hline $\begin{array}{l}\text { Di Maggio y Powell (1985) } \\
\text { Fombrun y Shanley (1990) } \\
\text { Fombrum (1996) } \\
\text { Robert y Dowling (2002) } \\
\text { Scott (2003) }\end{array}$ & $\begin{array}{l}\text { Institutional Theory } \\
\text { (teoría institucional) }\end{array}$ & $\begin{array}{l}\text { La ventaja sostenible de una empre- } \\
\text { sa depende de su capacidad para } \\
\text { manejar el contexto institucional y } \\
\text { sus recursos. }\end{array}$ \\
\hline $\begin{array}{l}\text { Barney (1991), (2001) } \\
\text { Amit y Shoemaker (1993) } \\
\text { Kreps y Wilson (1982) } \\
\text { Milgrom y Roberts (1982) } \\
\text { Shapiro (1982, 1983) } \\
\text { Allen (1984) } \\
\text { Weigelt y Camerer (1988) } \\
\text { Grant (1991) } \\
\text { Belkaoui y Pavlik (1992) } \\
\text { Hall (1992), (1993) } \\
\text { Celentani, Fudenberg, Levine, y } \\
\text { Pesendorfer (1996) } \\
\text { Oliver (1997) } \\
\text { Russo y Fouts (1997) } \\
\text { Vendelo (1998) } \\
\text { Balmer y Gray (1999) } \\
\text { Deephouse (2000) } \\
\text { Phelan y Lewin (2000) } \\
\text { Baden-Fuller y Ang (2001) } \\
\text { Roberts y Dowling (2002) } \\
\text { Anca E. Cretu and Roderick J. } \\
\text { Brodie (2009) }\end{array}$ & $\begin{array}{l}\text { Resource-based Theory } \\
\text { (teoría de los recursos de la firma) }\end{array}$ & $\begin{array}{l}\text { Está fundamentada en la ventaja } \\
\text { competitiva que tiene la empresa de } \\
\text { acuerdo con los recursos materiales } \\
\text { y humanos que posee. }\end{array}$ \\
\hline $\begin{array}{l}\text { Freeman (1984) } \\
\text { Wood y Jones (1995) } \\
\text { Mitchell, Agle, y Wood (1997) } \\
\text { Frooman (1999) } \\
\text { Jones y Wicks (1999) } \\
\text { Mahon (2002) }\end{array}$ & $\begin{array}{l}\text { Stakeholder Theory } \\
\text { (teoría de los públicos) }\end{array}$ & $\begin{array}{l}\text { Los públicos interesados pueden } \\
\text { perder o ganar por el éxito o el } \\
\text { fracaso de una empresa. }\end{array}$ \\
\hline
\end{tabular}




\begin{tabular}{|c|c|c|}
\hline $\begin{array}{l}\text { Goldberg y Hartwick (1990) } \\
\text { Rao (1994) } \\
\text { Shamsie (2003) } \\
\text { Rindova, Pollock y Hayward (2006) }\end{array}$ & $\begin{array}{l}\text { Social Construction Theory } \\
\text { (teoría de la construcción social) }\end{array}$ & $\begin{array}{l}\text { La reputación es producto de una } \\
\text { construcción social: } \\
\text { Los stakeholders comprenden las } \\
\text { señales estratégicas que emanan } \\
\text { de las empresas, las mismas que } \\
\text { buscan influir en los observadores. }\end{array}$ \\
\hline $\begin{array}{l}\text { Podolny (1993) } \\
\text { Stuart (2000) } \\
\text { Geletkanycz, Boyd and Finkelstein } \\
\text { (2001) } \\
\text { Gulati y Higgins (2003) } \\
\text { Rao, Monin y Durand (2003) } \\
\text { Rhee y Haunschild (2006) } \\
\text { Jensen y Roy (2008) }\end{array}$ & $\begin{array}{l}\text { Role Theory } \\
\text { (teoría de roles) }\end{array}$ & $\begin{array}{l}\text { La reputación es producto de la eva- } \\
\text { luación de los beneficios pasados } \\
\text { que cumplen con las expectativas } \\
\text { de rol asociadas con el status de } \\
\text { la persona. Se comprende a la } \\
\text { reputación como un mecanismo de } \\
\text { movilidad social. }\end{array}$ \\
\hline $\begin{array}{l}\text { Fombrun y Shanley (1990) } \\
\text { Fombrun (1996) } \\
\text { Fombrun y Van Riel (2004) } \\
\text { Gardberg y Fombrun (2006) }\end{array}$ & $\begin{array}{l}\text { Reputation, Performance and Intan- } \\
\text { gible Assets } \\
\text { (Reputación, rendimiento y activos } \\
\text { intangibles) }\end{array}$ & $\begin{array}{l}\text { La reputación está influenciada } \\
\text { por la publicidad, la rentabilidad, la } \\
\text { ciudadanía, la diversificación, y está } \\
\text { inversamente relacionada con el } \\
\text { riesgo financiero. }\end{array}$ \\
\hline Van Riel y Fombrun (2007) & $\begin{array}{l}\text { Corporate Communication \& } \\
\text { Reputation } \\
\text { (Comunicación Corporativa y } \\
\text { Reputación) }\end{array}$ & $\begin{array}{l}\text { Mirada integral de las teorías de co- } \\
\text { municación corporativa y la relación } \\
\text { con la reputación. }\end{array}$ \\
\hline $\begin{array}{l}\text { Abratt (1989) } \\
\text { Aaker (1991) } \\
\text { Bromley (1993) } \\
\text { Dowling (1993) } \\
\text { Balmer (1997) } \\
\text { Keller (1998) } \\
\text { Hatch, Schultz y Larsen (2000) } \\
\text { Brown et al. (2005) }\end{array}$ & $\begin{array}{l}\text { Brand, Identity and Culture } \\
\text { (marca, identidad y cultura) }\end{array}$ & $\begin{array}{l}\text { Las compañías construyen distintas } \\
\text { reputaciones y posiciones a través } \\
\text { de lo que manifiestan. }\end{array}$ \\
\hline $\begin{array}{l}\text { Abrahamson y Fombrun (1994) } \\
\text { Rindova y Fombrun (1999) }\end{array}$ & $\begin{array}{l}\text { Macro- Culture \& Cognitive Competi- } \\
\text { tive Advantage } \\
\text { (macro-cultura y ventaja competitiva } \\
\text { cognitiva) }\end{array}$ & $\begin{array}{l}\text { Las empresas habitan en ambientes } \\
\text { socioculturales de los que sacan la } \\
\text { legitimidad y que influyen para dife- } \\
\text { renciarse, atraer recursos y construir } \\
\text { ventaja competitiva. }\end{array}$ \\
\hline $\begin{array}{l}\text { Suchman (1985) } \\
\text { Deephouse / Carter (2005) } \\
\text { King y Whetten (2008) }\end{array}$ & $\begin{array}{l}\text { Legitimacy and Reputation } \\
\text { (legitimidad y reputación) }\end{array}$ & $\begin{array}{l}\text { La legitimidad hace hincapié en la } \\
\text { aceptación social que se deriva de } \\
\text { la adhesión a las normas sociales y } \\
\text { las expectativas, mientras que la re- } \\
\text { putación hace hincapié en las com- } \\
\text { paraciones entre las organizaciones. }\end{array}$ \\
\hline
\end{tabular}

Fuente: Elaboración propia a partir de la propuesta de Mahon (2002), Rindova et al. (2005) y Fombrun (2012) en The Oxford handbook of Corporate Reputation (2012).

Como se puede observar en la Tabla 2, los enfoques teóricos desde los cuales se ha estudiado el fenómeno de la reputación son diversos y, a pesar de la diferencia que existe entre cada uno de ellos, la gran mayoría coincide en que la reputación se caracteriza por ser: a) multidimensional; b) intangible; c) influyente en el individuo u organización que la posee; d) producto de una representación colectiva de acciones pasadas de la firma o el individuo; 
y (e) resultado de juicios acumulados de los públicos con relación a la organización o al individuo. $^{5}$

En este sentido, autores como Fomburn y Rindova (1996), citado en Fombrun y Van Riel (1997), sostienen que:

La reputación corporativa es una representación colectiva de las acciones pasadas de una empresa y el rendimiento que describe la capacidad de la empresa para entregar resultados valiosos a múltiples partes interesadas. Se mide la posición relativa de una empresa a nivel interno con los empleados y, externamente con sus grupos de interés, tanto en sus entornos competitivos e institucionales (Fombrun y Van Riel, 1997, 10).

Estos expertos también plantean seis áreas de estudio de la reputación que son: 1. económica, 2. estratégica, 3. organizacional, 4. sociológica, 5. contable y, 6. mercadotecnia.

La visión económica de la reputación se ha desarrollado a través del estudio de varias teorías, entre ellas: "Teoría de los costes de transacción, Teoría de juegos, Teoría de la eficacia organizativa, Teoría de la información, Teoría de agencia y la Teoría de los recursos y capacidades, tesis que han influido en su marco teórico" (Martínez y Olmedo, 2010, 62).

La Teoría de juegos ha sido de utilidad para el estudio de la reputación porque se la emplea en situaciones de toma de decisiones; procesos que son permanentes en la vida diaria de las personas y que ameritan una mayor reflexión en algunos casos mientras que, en otros, corresponden a ciertos hábitos o comportamientos ya adquiridos por el sujeto y manifestados a través de acciones automatizadas (Molina, Prada y Saavedra, 2007). Esta teoría sugiere que en función de la información que poseen los jugadores —la percepción que tienen de los otros- pueden establecer sus estrategias y movimientos, los cuales pueden generarles una reputación positiva o negativa (Weigelt y Camerer, 1988).

Mientras que, la Teoría de señales se fundamenta en la importancia de la asimetría ${ }^{6}$ de la información. Desarrollada en los años setenta por Spence (1974), citado en Morong $(2007,18)$, sugiere que: "Las señales del mercado son actividades o atributos de los individuos que, por diseño o accidente, alteran las creencias de otros individuos o les transmiten información a través del mercado". Es decir, que la información otorga al que la posee un poder de decisión más estratégico y estudiado e incluso con menor margen de error que al desinformado.

En relación con el comportamiento del sujeto, sus aciertos y desaciertos, así como la mirada e impresiones que tienen los otros sobre él (es decir, lo que piensan y lo que conocen sobre su persona), la Teoría de Señales no solo examina las señales emitidas sino también las que son interpretadas por los receptores pues indaga en el uso "de la retórica y su influencia en las percepciones de la contraparte" (Walker, 2010, 377).

De acuerdo con esta perspectiva, un ejemplo del análisis de la influencia que tienen las percepciones de la contraparte puede ser el examen de episodios relacionados con el auto-sacrificio público (e.g., hacer donaciones a obras de voluntariado, prescindir de algo propio para dárselo a otra persona o invertir el tiempo en causas benéficas) pues tiene conexión directa

$5 \quad$ E.g., Argenti y Forman, 2002; Caprioti, 2009; Corporate Excellence, 2012; De Quevedo, De la Fuente, Delgado, 2005; Dowling, 2001; Drobis, 2000; Fombrun, 1996; Fombrun, Gardberg y Sever, 2000; Fombrun y Van Riel, 1997; Fombrun y Van Riel, 2004; Fombrun y Van Riel, 2007; Goldberg et al., 2003; Gray y Balmer, 1998; López y Sebastián, 2009; Mahon, 2002; Martínez y Olmedo, 2009; Miles y Covin, 2000; Miles and Covin, 2002; Villafañe, 2003; Walker, 2010.

6 "La asimetría consiste en que diferentes personas conocen diferente información, la misma que les proporciona mayor o menor poder en la toma de decisiones de acuerdo con el tipo y cantidad de información que poseen sobre una determinada situación o persona" (Stiglitz, 2002, 469). 
con el estatus de la persona porque esos actos demuestran que el individuo está dispuesto a asumirlo todo por el bienestar común y, frente a los otros, será considerado como una persona con buena reputación por su generosidad (Griskevicius, Tybur y Bergh, 2010).

En el campo de estudio de la reputación personal la Teoría de Señales subraya que los individuos coexisten en los mercados de cambio en donde se relacionan con otros y les envían señales reales o potenciales. En virtud de esto, la reputación se convierte en una de esas señales que en ocasiones influye en los demás, en las impresiones que tienen los otros sobre el sujeto que posee reputación o sobre la organización a la que este individuo representa.

\section{Visión estratégica de la reputación}

El enfoque estratégico también corresponde a la ciencia económica y señala que la reputación es un activo intangible (Fombrun y Van Riel, 1997) y que "implícitamente direcciona las asignaciones de los recursos que las empresas deben hacer a través del tiempo para erigir barreras de reputación a la movilidad de los rivales" (Barney, 1986 citado en Fombrun y Van Riel, 1997, 7).

Mahon (2002), citando a varios autores ${ }^{7}$ afirma que la reputación per se no ha sido considerada como estratégica, sino que de ordinario se la cataloga como un recurso que genera ventaja para la empresa frente a la competencia o circunstancias adversas. Es decir, este autor propone que se reconozcan los activos intangibles y los elementos que caracterizan a la organización (i.e. recursos materiales, recursos humanos, éxitos, reconocimiento social, rentabilidad de la organización, etc.) y que se los emplee en beneficio de la organización. Esta premisa trasladada al plano individual legitima a la reputación como un activo personal.

\section{Enfoque organizacional de reputación}

Desde este ámbito se ha estudiado a la reputación en función de la cultura empresarial, las relaciones de los empleados, sus percepciones y motivaciones. La reputación se convierte en un reflejo externo de la cultura organizacional de la empresa, es la manifestación de cómo ven los empleados a la corporación y cómo se sienten trabajando en ella. Forman parte importante también los valores y la identidad de la organización.

En esta disciplina también se encuadra la Teoría de los Stakeholders que se enfoca en los diversos grupos de interés con los que interactúa la organización. Los inversores, clientes y empleados son generalmente vistos como los principales interesados en razón de su poder y legitimidad, así como por su capacidad para responder demandas urgentes de las empresas (Fombrun, 2012). Es decir, se trata del estudio de la reputación de qué o de quién y para quién. Esta teoría propone que la construcción de la reputación está basada en un conjunto de percepciones internas o externas en función de los públicos que se estudian, de ahí que la concibe como un concepto colectivo (Walker, 2010).

$7 \quad$ Baden-Fuller y Ang, 2001; Balmer y Gray, 1999; Barney, 1991, 2001; Belkaoui y Pavlik, 1992; Celentani, Fudenberg, Levine y Pesendorfer, 1996; Clark y Montgomery, 1998; Deephouse, 2000; Dutton y Dukerich, 1991; Fiol, 2001; Freeman, 1984; Grant, 1991; Hall, 1992, 1993; Kotha, Rajgopal, y Rindova, 2001; Milgrom y Roberts, 1982; Phelan y Lewin, 2000; Oliver, 1997; Rao, 1994; Russo y Fouts, 1997; Vendelø, 1998; Weigelt y Camerer, 1988, Cuevas y Porter, 1977. 


\section{Marco sociológico y psicosocial de la reputación}

Dentro de los estudios sociológicos, los expertos suelen denominar a la reputación como prestigio o estima porque se la observa en función del estatus que le otorga al individuo en la estratificación social (e.g., Fombrun y Zajac, 1987; Shenkar y Yuchtman-Yaar, 1997). El estatus se considera aquí como el rol social, que no es únicamente la puesta en marcha de la posición que ocupa el individuo u organización dentro de la sociedad, sino también el comportamiento del sujeto o la institución frente a la función que debe cumplir socialmente (Berguer, 2014).

De ahí que la Teoría de rol (role theory) permite inferir la reputación del individuo u organización a través de la evaluación del desempeño social que tiene porque involucra valores, creencias, normas, etc. Al ser un elemento en constante desarrollo, producto de creencias, narraciones evolutivas y expectativas que se van construyendo y modificando a partir de un diálogo entre la empresa y los públicos en el tiempo, como ya se dijo antes, es indispensable que se estudie considerando el contexto en que se desarrolla. Esto también permite conocer las diversas posiciones que ocupa el individuo en función de sus comportamientos dentro de un tiempo y espacio determinados (e.g., Aula y Mantere, 2012; Jensen et al., 2012).

Montgomery (1998) también coincide con la importancia de estudiar los roles y la evaluación del comportamiento de los individuos en relación con el contexto. Este autor sostiene que los roles permiten identificar si se ajustan (o no) las conductas a las normas sociales establecidas $y$, por ende, ayuda a determinar si son positivas o negativas las actuaciones del sujeto u organización. En este sentido, se reconoce a la reputación como un elemento que permite predecir los comportamientos futuros con base a la evaluación de la conducta pasada del sujeto o de la organización (Jensen et al., 2012).

La reputación también se ha estudiado desde la perspectiva psicosocial a partir del enfoque de la Teoría de la Influencia social y Gestión de Impresiones. Esta propuesta denominada como self-presentation (autopresentación) consiste en un "proceso mediante el cual los individuos tratan de controlar las impresiones que los otros crean sobre ellos" en las relaciones sociales (Learly y Kowalski, 1990:34). En estos intercambios, las personas usualmente buscan mostrar una imagen positiva frente a los otros. En este sentido, el comportamiento del sujeto puede ser consciente e intencionado con la finalidad de ser "percibido, evaluado y tratado lo más favorablemente posible" por los demás (López et al., 2013:63).

Este supuesto teórico de gestión de impresiones no es un tema nuevo en los estudios relacionados con el comportamiento humano y la psicología social; surge a partir de los estudios realizados por el sociólogo Ervin Goffman (1981) quien pone en evidencia que las prácticas cotidianas de los sujetos son experimentos constantes de su legitimidad y comprobación continua de su reputación.

\section{Mirada contable de la reputación}

Desde el ámbito contable, los investigadores expertos en el área plantean que no existen formas de medición de las inversiones que realizan las empresas en sus marcas, capacitación del personal y desarrollo de investigación en las organizaciones. A pesar de la importancia de estas inversiones, no se logra cuantificar el dinero que se destina a estos activos intangibles, ni los ingresos que se obtienen por ellos como es el caso de la reputación 
de una marca. En este sentido, considerar la reputación como un activo intangible dificulta la identificación de cuáles son los elementos que la componen, porque su desarrollo en el tiempo es producto de una serie de intercambios y relaciones entre los interesados directos y la organización en diversos contextos y momentos (Mahon, 2002; Rao, 1994).

\section{Mercadotecnia y reputación}

Como se manifestó al inicio, otra área desde la que se indaga sobre reputación es el marketing que tiene como principal sujeto de estudio a los consumidores, a quienes ofrecen sus productos a través de estrategias de marca y, mediante un logo, una campaña, el boca a boca, etc., dan a conocer la empresa y los productos o servicios que esta ofrece. Esta visión mercadotécnica, presenta a la reputación como la imagen de marca a la que el consumidor le atribuye un significado que lo lleva a rechazarla o aceptarla.

En resumen, este breve acercamiento al objeto de estudio de la reputación corporativa - que han tenido varias escuelas y teorías de los más diversos ámbitos-, da cuenta del carácter multidimensional que frecuentemente se le adjudica a la reputación. Asimismo, acredita que el estudio de la reputación desde el enfoque organizacional ha sido y continúa siendo amplio y necesario, porque es un bien intangible que aporta muchos beneficios a la empresa.

\section{Aporte científico a la reputación personal}

El dinamismo inherente a la naturaleza de la reputación es afín a la propuesta que realiza Ervin Goffman (1981, 133), cuando en su obra Asyllums hace alusión a la carrera moral del paciente mental y señala que "la carrera se ha reservado, tradicionalmente, para quienes aspiran a escalar las sucesivas etapas que presenta una profesión honorable". Además, la define como "cualquier trayectoria social recorrida por cualquier persona en el transcurso de la vida". Este supuesto de Goffman (1981) — sobre la carrera moral del individuo- concuerda también con la propuesta que autores como Becker (2009) sugieren sobre la construcción de la reputación cuando reconocen que su naturaleza es cambiante y que evoluciona a lo largo del tiempo.

Otros académicos interesados en el estudio de la organización, las relaciones humanas y la administración, plantean que la información existente sobre el tema es escasa a pesar de su relevancia (Zinko et al., 2012). Para Ferris et al. (2003), la obra más completa sobre reputación personal es la propuesta que hace Bromley (1993) en su libro Reputation, image and impression management, donde expone que la reputación es un tipo de influencia "que incide en la autoestima, la identidad social, el comportamiento individual y las interacciones sociales (...), señala que la persona tiene tantas reputaciones como número de grupos sociales con los que interactúa" (Bromley citado en Ferris et al., 2003, 9). Esta afirmación también se relaciona con el planteamiento sociológico de la Teoría de rol, formulado por Jensen et al. (2012) —expertos en reputación, estrategia y Teoría organizacional—, que conciben a la reputación como un componente diferenciador de los individuos dentro de un mismo estatus, es decir, que las personas puedan tener múltiples reputaciones con la misma audiencia.

Este hallazgo de Bromley (1993) — sobre las múltiples reputaciones que tiene el individuo— también coincide con el aporte teórico que realizan Davies y Harré (2007), estudiosos en psicología social, que sostienen que una persona puede ocupar diferentes posiciones en la sociedad, y esa posición puede ser a la vez ocupada por varias personas. Al igual que 
Bromley (1993), ambos autores señalan que estas posiciones están enmarcadas en función de la interacción verbal del individuo frente a otros.

La Teoría de posicionamiento acuñada por los estudiosos posestructuralistas, Davies y Harré (2007), así como la propuesta de múltiples reputaciones de Bromley (1993), se relacionan con la Teoría de la identidad social la cual define al individuo como un sujeto compuesto por varias identidades, las mismas que reflejan diversas posiciones que la persona tiene dentro de los múltiples grupos con los que interactúa (Tajfel y Turner, 1979). Lo dicho anteriormente también evidencia las opiniones y creencias que los individuos tienen sobre sí mismos. Además, las validaciones de estas autoidentidades preconcebidas se refuerzan cuando las situaciones sociales coinciden con las identidades (Zinko y Rubin, 2015, 226).

Retomando las diversas acepciones de reputación personal, varios autores (e.g. Zinko et al., 2012) la definen como una "construcción cognitiva social", concepción que coincide con las propuestas que desde la sociología se plantean sobre la reputación corporativa. Al igual que esta, varios académicos sostienen que posee una estrecha relación con otros conceptos como: el estatus, la imagen, la fama, la celebridad, el pedigree, la legitimidad, la credibilidad, el branding y el manejo de impresiones. En el cuadro que sigue, se explican cada uno de estos conceptos de acuerdo a lo estudiado por: Greyser, 1999; Rao, 1994; Rindova, Pollock y Hayward, 2006; Vedder y Wachbroit, 2003.

\section{Tabla 3. Conceptos relacionados con la Reputación Personal}

\begin{tabular}{|c|c|c|}
\hline Concepto & Definición & Autores \\
\hline Estatus & $\begin{array}{l}\text { Posición que una persona tiene dentro de algún grupo u } \\
\text { organización y el prestigio que dicha posición le otorga. }\end{array}$ & $\begin{array}{l}\text { Weber citado en Berger (2012) } \\
\text { Dafoe, Renshon y Huth (2014) }\end{array}$ \\
\hline Imagen & $\begin{array}{l}\text { Conjunto de rasgos que caracteriza ante la sociedad a } \\
\text { una persona o entidad. Producto de una construcción } \\
\text { social. Percepción sobre una persona en función de la } \\
\text { apariencia que esta proyecta sobre sí misma, mediante } \\
\text { su conducta, fachada, lenguaje, etc., en un contexto de- } \\
\text { terminado y en relación con un grupo social específico. }\end{array}$ & $\begin{array}{l}\text { Berger y Luckman (1986) } \\
\text { Robert, P.W. and Dowling, G.R. } \\
(1997)\end{array}$ \\
\hline Fama & $\begin{array}{l}\text { Renombre, opinión pública que se tiene de una per- } \\
\text { sona. El reconocimiento otorgado por una situación } \\
\text { específica. }\end{array}$ & $\begin{array}{l}\text { Barcia (1882) } \\
\text { Rindova et al. (2006) } \\
\text { Zinko et al. (2015) }\end{array}$ \\
\hline Celebridad & $\begin{array}{l}\text { Popularidad intencionada por el reconocimiento mediá- } \\
\text { tico o una situación específica. }\end{array}$ & $\begin{array}{l}\text { Rindova et al. (2006) } \\
\text { Zinko y Rubin (2015) }\end{array}$ \\
\hline Pedigree & Similar al estatus. El valor del poder social. & Zinko y Rubin (2015) \\
\hline Legitimidad & $\begin{array}{l}\text { Juicio moral de la actuación de una persona u organi- } \\
\text { zación frente a una serie de normas y principios social- } \\
\text { mente aceptados. }\end{array}$ & $\begin{array}{l}\text { Whetten, D., Foreman, P., \& } \\
\text { Dyer. W. G. (2014) } \\
\text { Zinko y Rubin (2015) }\end{array}$ \\
\hline Credibilidad & Legitimidad en la personalidad. & $\begin{array}{l}\text { Ferris et al. (2003) } \\
\text { Zinko y Rubin (2015) }\end{array}$ \\
\hline Branding & $\begin{array}{l}\text { Construcción de la marca. Visión y cultura, personali- } \\
\text { dad, etc., todo lo que refleje la imagen real y deseada } \\
\text { de las partes interesadas. }\end{array}$ & Harris \& Chernatony (2001) \\
\hline $\begin{array}{l}\text { Manejo de } \\
\text { impresiones }\end{array}$ & Gestión estratégica de las actuaciones personales. & Leary y Kowalski (1990) \\
\hline
\end{tabular}

Fuente: Elaboración propia a partir de varios autores. 
Sin embargo, Ferris et al., (2003) sostienen que la reputación en su totalidad, siendo de naturaleza perceptual y subjetiva, es más que una "realidad socialmente construida" y está definida por el "ojo del espectador", es decir, que no es de carácter objetivo. Por esto, la reputación no se concibe como un producto de la instantaneidad sino de una acumulación temporal de comportamientos y acciones realizadas por el sujeto que, en ocasiones, pueden presentarse en forma de "señales" (Zinko R. F., 2012, 157). Estas señales permiten inferir cuáles serán los futuros movimientos de la persona, o cuál es su patrón de comportamiento frente a determinadas situaciones, pues aportan información valiosa sobre el individuo.

Este presupuesto de Ferris et al. (2003) coincide con el trabajo de Bromley (2001) quien define la reputación como una imagen colectiva, producto de las opiniones acerca de una persona, es decir, los diversos juicios que surgen a partir de cada uno de los grupos con los que se relaciona el individuo. Por consiguiente, cada persona tiene una reputación distinta, en concordancia con las impresiones del grupo de interés que la evalúa.

Como ya se dijo, un aporte contemporáneo relevante de la investigación en el tema de reputación personal son los estudios realizados por Zinko et al. (2012). Sus conclusiones refuerzan las siguientes nociones: 1 . que la reputación del individuo influye en la reputación de la organización; 2 . que la reputación del sujeto se desarrolla en el tiempo; y, 3. que la reputación está determinada por un contexto. Incluso, ciertos estudiosos (e.g., Hollander citado en Ferris et al., 2003; Zinko et al., 2007; Zinko et al., 2015) afirman que la reputación es producto de la desviación del comportamiento de los individuos frente a las normas establecidas en el entorno que interactúan, ya sea de tipo organizacional, social, etc.

Los aportes realizados por Zinko et al. (2012), ${ }^{8}$ a partir de los estudios sobre la construcción de la reputación del individuo dentro de la organización, dan cuenta de que el capital humano, la eficacia social y el tiempo son elementos importantes en la construcción de la reputación personal. Para estos autores, el capital humano comporta el conocimiento y las habilidades que tiene el sujeto $y$, además, son elementos que otorgan reconocimiento público a la persona. Asimismo, denotan que este componente (capital humano) se relaciona con la raza, el género y la edad, variables constitutivas de su perfil sociodemográfico.

En relación con la eficacia social, Ferris et al. (2005) y Zinko et al. (2012) señalan que consiste en que el individuo aplique sus conocimientos en busca de la efectividad laboral, el cumplimiento de los objetivos y metas personales y de la organización. Asimismo, esta persona se caracteriza por influir en el equipo de trabajo para que este sea proactivo y eficaz.

Otro factor importante que plantean Zinko et al. (2012) y que coincide con otras propuestas, como ya se mencionó, es el tiempo. Ellos sostienen que está íntimamente ligado con la naturaleza de la reputación porque las acciones del individuo se sujetan a un período y acontecen en uno o varios momentos de su historia personal (Zinko, 2012). Por lo tanto, la reputación no es estática sino dinámica porque aparece, crece y cambia con el tiempo (Ferris, 2014).

Entre los autores recientes que han estudiado el tema está Jácome (2016), que estudia la reputación personal del DirCom español. Esta experta señala que la Teoría de gestión de impresiones ${ }^{9}$ antecede a la reputación personal, es decir, que esta última es consecuencia de la gestión de las impresiones que realiza un individuo. Asimismo, manifiesta que la diferencia entre ambos constructos (impression management y reputación personal) se da

8 Para su investigación, estos expertos realizan observaciones del sujeto de estudio en su sitio de trabajo y la relación que mantiene con sus colegas dentro de la organización.

9 Teoría de la Gestión de Impresiones, que se define como el proceso de la gestión de las impresiones para proyectar imágenes favorables (Jácome, 2016, 69). 
porque la gestión de las impresiones está determinada por los individuos, pues ellos son los que deciden cómo mostrarse a través de su comportamiento y forma de hablar para influir en los demás. En cambio, la reputación es otorgada por los demás y no puede ser controlada por la persona. "Dicho de otro modo, mientras que la teoría de impression management es provocada, la reputación personal es otorgada" (Jácome, 2016, 70).

A partir del análisis de los dos conceptos mencionados reputación personal y gestión de impresiones Jácome (2016) define la reputación personal como:

La consideración que se otorga a una persona basada en una serie de percepciones construidas a lo largo del tiempo fruto de la observación — directa o indirecta (a través de otros) - de la imagen que proyecta, de sus características personales, de sus logros y de la historia de su comportamiento en los distintos entornos (...) Asimismo, continúa diciendo que la reputación es 'fruto de una ponderación del juicio realizada conforme a la huella que ha dejado el comportamiento de una persona a lo largo del tiempo en quien la observa, directa o indirectamente'. Y afirma que es producto de la 'influencia social', la 'personalidad del individuo', la 'integridad de sus comportamientos', ${ }^{10}$ el 'desempeño y resultados obtenidos con sus comportamientos individuales' (2016, 82-84).

A partir de esta propuesta, la autora evidencia la existencia de una analogía entre las dimensiones de la reputación personal (e.g., social, desempeño e integridad) y las dimensiones de la Teoría de gestión de impresiones (e.g., social, organizacional y ética), en la que señala que la dimensión "ética (e.g., el carácter /integridad)" es la más importante porque "hace que las personas sean percibidas como íntegras y fiables en su comportamiento porque se observa una coherencia entre lo que esa persona es, sus palabras y sus acciones-", lo que presupone la confianza por parte de los otros, su credibilidad y, en definitiva, una reputación positiva (Jácome, 2016, 86-87).

Un estudio más reciente es el de Sotillo, en este señala que la reputación del Chief Executive Officer (CEO) es equivalente a la reputación personal porque es,

El reconocimiento que los miembros de una comunidad realizan sobre un conjunto de características de una persona, su comportamiento a lo largo del tiempo y las imágenes que se proyectan sobre el sujeto, habiendo sido percibido por cualquier tipo de fuente de información por parte de esa comunidad concreta. (Sotillo, 2017, 77)

Esta experta también sostiene que los conceptos de marca del CEO y reputación del CEO son distintos, pero están relacionados. Porque para los expertos en reputación "la marca es un proceso de gestión para alcanzar la reputación deseada (...) debe estar conectada con la visión, misión, los valores corporativos y las claves del proyecto empresarial" (86).

Y propone la siguiente definición de reputación del CEO:

Reconocimiento que los stakeholders hacen de su capacidad para generar valor de manera consistente a lo largo del tiempo en la compañía de la que están al frente, en función de su histórico de comportamiento como persona, profesional y representante corporativo (Sotillo, 2017, 114).

Es decir, que logra "reducir la incertidumbre sobre el comportamiento futuro del ejecutivo, y por ende de la organización que lidera, tiene la capacidad de generar confianza e impactar positivamente en el rendimiento de la organización" (114). En este sentido, se puede afirmar que la reputación del CEO impacta directamente en la reputación de la organización.

10 Actuar de acuerdo a sus principios y valores (Jácome, 2015, 84). 


\section{CONCLUSIONES}

Hasta aquí se ha transitado exploratoriamente el camino que los académicos han recorrido sobre el estudio de la reputación. Se han explicado las escuelas desde dónde se ha estudiado el concepto y los términos con los que guarda relación como: fama, imagen, identidad, estatus y legitimidad. Además, se han repasado los aportes de expertos en el tema desde distintos ámbitos como: organizacional, relaciones humanas y administrativo. Con esta revisión teórica se puede concluir que la reputación es de naturaleza perceptual y que se construye mediante la acumulación temporal de comportamientos y acciones realizadas por la organización o el sujeto, situados en un tiempo y un espacio determinados, es decir, un contexto.

\section{BIBLIOGRAFÍA}

Barcia, R. (1894). Primer diccionario general etimológico de la lengua española (vol. 5). Barcelona, España: Seix-Editor.

Barnett, M., Lafferty, J. M., y Jermier, B. A. (2006). Corporate reputation: The definitional landscape. Corporate reputation review, 9 (1): 26-38.

Becker, G. S. (2009). Human capital: A theoretical and empirical analysis, with special reference to education. University of Chicago press.

Berger, A. A. (2014). Técnica de análisis de medios de comunicación (1. ${ }^{a}$ ed., 1). (U. C. Grande, Ed., C. T. Chiriboga, A. M. Tanca, y M. C. Noboa, Trad.) Guayaquil, Ecuador: Universidad Casa Grande.

Bromley, D. B. (1993). Reputation, image and impression management. John Wiley \& Sons.

Davies, B., y Harré, R. (2007). Posicionamiento: La producción discursiva de la identidad. Athenea digital: Revista de pensamiento e investigación social, 12: 242-259.

Emler, N. (1990). A social psychology of reputation. European Review of Social Psychology, 1 (1): 171-193.

Ferris, G. R., Blass, F. R., Douglas, C., Kolodinsky, R. W., Treadway, D. C., y Greenburg, J. (2003). Personal reputation in organizations. Organizational Behavior: A Management Challenge, 201.

Ferris, G. R., Treadway, D. C., Kolodinsky, R. W., Hochwarter, W. A., Kacmar, C. J., Douglas, C., \& Frink, D. D. (2005). Development and validation of the political skill inventory. Journal of management, 31(1): 126-152.

Ferris, G. R., Harris, J. N., Russell, Z. A., Ellen III, B. P., Martinez, A. D., y Blass, F. R. (2014). The role of reputation in the organizational sciences: A multilevel review, construct assessment, and research directions. In Research in personnel and human resources management (241303). Emerald Group Publishing Limited.

Fombrun, C. (1996). Reputation: Realizing Value from The Corporate Image. Boston, EE. UU.: Harvard Business School Press.

Fombrun, C. J., y Van Riel, C. B. (1997). The reputational landscape. Corporate reputation review, 1 (2): 5-13.

Fombrun, C. I. (2012). The Building Blocks of Corporate Reputation: Definitions, Antecedents, Consequences. En O. U. Press, y M. P. Barnett (Ed.), The Oxford Handbook of Corporate Reputation (pág. 502). Oxford: Oxford University Press.

Goffman, E. (1981). La presentación de la persona en la vida cotidiana (Vol. 60). (Hildegarde B. Torres Perrén y Flora Setaro, Trad.) Buenos Aires, Buenos Aires, Argentina: Amorrortu.

Goffman, E. (2001). Internados: Ensayos sobre la situación social de los enfermos mentales. Buenos Aires, Argentina: Amorrourtu. 
Griskevicius, V., Tybur, J. M., y Van den Bergh, B. (2010). Going green to be seen: status, reputation, and conspicuous conservation. Journal of Personality and Social Psychology, 98 (3): 392403.

Jácome López, R. (2016). La impression management aplicada a la construcción de reputación personal y en las organizaciones: el caso del dircom (Doctoral dissertation).

Jensen, M., Kim, H., y Kim, B. K. (2012). Meeting expectations: A role-theoretic perspective on reputation. En M. L. Barnett, The Oxford handbook of corporate reputation, 140-159. Oxford, UK: Oxford University Press.

Leary, M. R., \& Kowalski, R. M. (1990). Impression management: A literature review and two-component model. Psychological Bulletin, 107 (1): 34-47.

López, R. J., Bañón Gomis, A. J., Parra, M. G., y Darós, L. C. (2013). Influencia de la teoría de Impression Management en los comportamientos que afectan a la relación entre personas y organizaciones. Working Papers on Operations Management, 4 (1): 9-22. https://doi. org/10.4995/wpom.v4i1.1488.

Mahon, J. F. (2002). Corporate reputation: A research agenda using strategy and stakeholder literature. Business \& Society, 41 (4): 415-445.

Martínez, I., y Olmedo, I. (2010). Revisión teórica de la reputación en el entorno empresarial. Cuadernos de Economía y Dirección de la Empresa, 13 (44): 59-77.

Molina, Y. P., Prada, R. B., y Saavedra, O. R. (2007). Allocation of transmission loss cost using game theory. In Power Tech, 407-412. Lausanne: IEEE.

Montgomery, J. (1998). Toward a Role-Theoretic Conception of Embeddedness. American Journal of Sociology, 104 (1), 92-125.

Morong, C. (2007). La intersección entre señales económicas y símbolos míticos. Revista de economía institucional, 9 (16): 15-33.

Rao, H. (1994). The social construction of reputation: Certification contests, legitimation, and the survival of organizations in the American automobile industry: 1895-1912. Strategic Management Journal, 15(S1): 29-44.

Rindova, V. P., Williamson, I. O., Petkova, A. P., y Sever, J. M. (2005). Being good or being known: An empirical examination of the dimensions, antecedents, and consequences of organizational reputation. Academy of Management Journal, 48(6): 1033-1049.

Sotillo Fraile, S. (2017). La gestión profesional de la reputación del CEO como elemento generador de valor para las organizaciones (Doctoral dissertation, Universitat Jaume I).

Walker, K. (2010). A Systematic Review of the Corporate Reputation Literature: Definition, measurement, and theory. Corporate Reputation Review, 12 (4): 357-387.

Weigelt, K., y Camerer, C. (1988). Reputation and Corporate Strategy: A Review of Recent Theory and Applications. Strategic Management Journal, 9 (5): 443-454.

Tajfel, H., y Turner, J. C. (1979). An Integrative Theory of Intergroup Conflict. In W. G. Austin, \& S. Worchel (Eds.), The Social Psychology of Intergroup Relations: 33-47. Monterey, CA: Brooks/Cole.

Zinko, R., Ferris, G. R., Blass, F. R., y Dana Laird, M. (2007). Toward a theory of reputation in organizations. Research in Personnel and Human Resources Management:163-204..

Zinko, R., Ferris, G. R., Humphrey, S. E., Meyer, C. J., y Aime, F. (2012). Personal reputation in organizations: Two-study constructive replication and extension of antecedents and consequences. Journal of Occupational and Organizational Psychology, 85 (1): 156-180.

Zinko, R., y Rubin, M. (2015). Personal reputation and the organization. Journal of Management \& Organization, 21 (2): 217-236. 